\title{
Investigation and management of Clostridium difficile colonisation in a paediatric oncology unit
}

\author{
Ildikó Schuller, Vaskar Saha, Lin Lin, Judith Kingston, Tim Eden, Soad Tabaqchali
}

\begin{abstract}
Little is known about Clostridium difficile infection in children with cancer but a presumed outbreak has previously been described. The carriage rate before admission to hospital and morbidity is reported to be high, especially in younger children. The prevalence of $C$ difficile infection on a paediatric oncology ward was monitored from June 1991 to May 1992. Twenty eight $(13 \%)$ of 214 children were found to be infected. Though the temporal distribution suggested an outbreak, polyacrylamide gel electrophoresis identified several different types. Unlike previous reports, infection appeared to be possibly endogenous or possibly environmental in origin rather than due to cross infection; the morbidity was low and age was not a determinant for infection. The duration of hospital stay and the primary diagnosis were found to be determinants for infections, those with lymphoid malignancies being at the highest risk. The diagnostic category at greatest risk were those most intensively treated, with protracted neutropenia and prolonged antibiotic exposure. Early identification of cases and prompt institution of simple control measures will prevent cross infection. It is therefore important that diarrhoea is not accepted as a normal symptom of cancer chemotherapy and stool specimens are sent for full bacteriological and viral investigation.

(Arch Dis Child 1995; 72: 219-222)
\end{abstract}

Keywords: Clostridium difficile, cancer.

The early and empiric use of antibiotic regimens in children with chemotherapy induced febrile neutropenia has radically decreased the morbidity and mortality previously associated with such treatment. ${ }^{1}$ Antibiotic regimens in different hospitals are tailored for locally prevalent organisms but, in most cases, consist of a third generation cephalosporin and an aminoglycoside with or without a penicillinase resistant penicillin. The broad spectrum activity of such combinations may, especially in those immunosuppressed, predispose to other infections. While fungal infections are frequent, the commonest bacterial offender is Clostridium difficile, the causative agent of pseudomembranous colitis and antibiotic associated colitis and diarrhoea. ${ }^{2}$ Children with cancer may be at a particular risk for developing $C$ difficile infection. The antibiotics whose prior use have been implicated in $C$ difficile enteritis include those most commonly used for treating febrile neutropenic patients including penicillins, cephalosporins, trimethoprim-sulfamethoxazole, and aminoglycosides. ${ }^{2}$ Additionally, cytotoxic drugs such as methotrexate may predispose to gut colonisation with $C$ difficile. ${ }^{2}$ Although studies have been carried out on the epidemiology and acquisition of $C$ difficile in adult patients with haematological malignancies, ${ }^{34}$ little information is available on $C$ difficile infection in children with cancer. There is one report of an outbreak in a paediatric oncology unit in UK, based on identification of toxin in stool and attributed to a change in the antibiotic policy. ${ }^{5}$ However, at least four different strains of $C$ difficile are capable of producing toxin 4 and therefore the conclusions of this study are seriously flawed. Isolation and identification of toxicogenic and non-toxicogenic strains are essential for epidemiological studies of patterns of cross infection. ${ }^{6}$ Another study suggests that children with malignant disorders have a high rate of infection with the organism even before chemotherapy or antimicrobial treatment. ${ }^{7}$ The authors attribute considerable morbidity to $C$ difficile infection in these children especially in the younger age group. In this paper, we discuss the lessons learned by studying the pattern and prevalence of $C$ difficile infection in a paediatric oncology unit over a one year period.

\section{Patients and methods}

The prevalence of $C$ difficile in stool cultures obtained from children with cancer admitted to a paediatric oncology unit was monitored over a one year period from June 1992 to May 1993. In our unit, stool cultures are sent routinely from all children with febrile neutropenia and any inpatient with diarrhoea. For the purposes of this study, diarrhoea was defined as an alteration in normal bowel pattern with the passage of three or more consecutive unformed stools within a 24 hour period. Infection with $C$ difficile was defined as isolation of the organism on faecal culture from patients with diarrhoea. Faecal specimens were plated onto cycloserine cefoxitin fructose agar and incubated anaerobically for 48 hours. Suspect colonies were identified as $C$ difficile by distinctive colonial morphology and smell of p-cresol, apple green fluorescence under long wave ultraviolet light ( $366 \mathrm{~nm}$ ) and the pattern of the volatile fatty acids, with the production of a major peak of isocaproic acid by gas liquid chromatography. Typing of the $C$ difficile strains to identify toxin and non-toxin producing strains was carried out using polyacrylamide gel electrophoresis (PAGE) of 


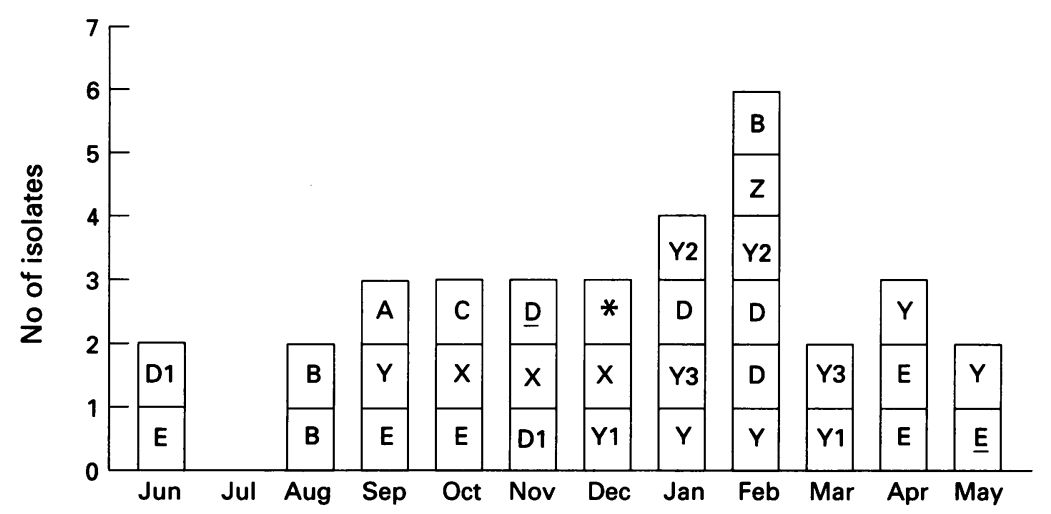

Radio-PAGE type of $\mathrm{C}$ difficile isolated from stool cultures from patients admitted to a paediatric oncology ward during Fune 1992 to May 1993. A total of 33 isolates were made from 28 children. $A, B, C, D, E, X, Y, Z$, and the radio-PAGE types; * is a strain that died before it could be typed. Y1, Y2, Y3, and D1 refer to the children who had a recurrence of infection with the same strain. $\underline{D}$ and $\underline{E}$ refers to the one child who was infected with two different strains six months apart.

${ }^{35}$ S-methionine labelled proteins (radioPAGE) as previously described. ${ }^{8-10}$

Patients were subsequently grouped into two categories according to duration of hospital stay. 'Short term' cases were those who stayed for less than 24 hours and were admitted for chemotherapy or investigation. 'Long term' cases stayed for a minimum of four days and were admitted for chemotherapy, febrile neutropenia, or terminal care. Both categories of patients shared all the ward facilities and, except for the very young, were not segregated. All children with $C$ difficile infection had serial monthly stool cultures until three successive cultures were negative.

To try to determine the risk factors for $C$ difficile infection, the age, gender, duration of hospital stay, and primary oncological diagnosis were compared with those of all uninfected children admitted during the same time period.

Statistical analysis included the use of the $\chi^{2}$ test and odds ratio where appropriate and a $p$ value of $<0.05$ was regarded as significant.

\section{Results}

During the one year study period, $C$ difficile was identified in 33 faecal specimens obtained from $28(13 \%)$ of 214 children with cancer

Table 1 Characteristics of children with cancer admitted to a paediatric oncology unit from fune 1992 to May 1993 according to the presence or absence of $\mathrm{C}$ difficile in faecal culture $(n=214)$

\begin{tabular}{lcc}
\hline & $\begin{array}{c}\text { C difficile } \\
\text { positive } \\
(n=28)\end{array}$ & $\begin{array}{l}\text { C difficile } \\
\text { negative } \\
(n=186)\end{array}$ \\
\hline Sex & 14 & 104 \\
$\quad$ Male & 14 & 82 \\
Female & & \\
Age (months) & $8-203$ & $0-247$ \\
$\quad$ Range & $58(49)$ & $76(63)$ \\
Mean (SD) & 2 & 98 \\
Day cases & 26 & 88 \\
Inpatients & 18 & 69 \\
Diagnoses & 4 & 64 \\
Leukaemia/lymphoma & 1 & 14 \\
Retinoblastoma & 2 & 13 \\
Central nervous system tumours & 3 & 7 \\
$\quad$ Neuroblastomas & 0 & 8 \\
Rhabdomyosarcomas & 0 & 7 \\
$\quad$ Bone tumours & 0 & 4 \\
$\quad$ Others & & \\
Benign tumours & & \\
\hline
\end{tabular}

Table 2 Antimicrobial treatment used in 28 children with $\mathrm{C}$ difficile infection

\begin{tabular}{ll}
\hline & No of children \\
\hline Symptomatic & 2 \\
Oral metronidazole & 5 \\
Intravenous metronidazole & \\
Oral metronidazole and & 2 \\
oral vancomycin & \\
Intravenous metronidazole & and oral vancomycin \\
$\quad$ No treatment & 1 \\
Asymptomatic & 9 \\
Oral metronidazole & 1 \\
No treatment & 8 \\
\hline
\end{tabular}

admitted to the paediatric oncology ward at St Bartholomew's Hospital, London. The number of isolates per month and the respective radio-PAGE is shown in the figure. In four children, $C$ difficile of the same type was recovered from stool samples taken at least one month apart with at least one normal stool culture during the intervening period. One child was reinfected with a different type six months after the first infection.

Of the 214 children, 100 (47\%) were admitted as day cases. Two $(2 \%)$ of the short term cases and $26(22 \%)$ of the 114 long term admissions were found to be infected with $C$ difficile. Therefore, the relative risk of infection for those admitted long term was 14 times higher than those admitted for a short term $\left(\chi^{2}=18 \cdot 5, \mathrm{df}=1, \mathrm{p}=<0 \cdot 005,95 \%\right.$ confidence limits $=3 \cdot 2$ and 49 , odds ratio $=14 \cdot 5$ ).

Table 1 shows the distribution according to age, gender, and primary oncological diagnosis in those infected and uninfected. Neither age nor gender were predictive for $C$ difficile infection. Acute leukaemia or lymphoma were diagnosed in $18(67 \%)$ of the 28 infected and $69(38 \%)$ of the 186 uninfected children. Children in this diagnostic category therefore appeared to be at a higher risk of infection with $C$ difficile $\left(\chi^{2}=6.4, \mathrm{df}=1, \mathrm{p}=<0.05,95 \%\right.$ confidence limits $=1 \cdot 2$ and $7 \cdot 6$, odds ratio $=3$ ). The numbers of patients in other diagnostic categories are too small for valid statistical comparison. Twenty two were infected within the first three months and two each at five, 10, and more than 12 months after diagnosis.

Nineteen $(68 \%)$ were severely neutropenic (absolute neutrophil count $<0.5 \times 10^{9} /$ ) at the time $C$ difficile was isolated from their stools. Nineteen of the 28 children had symptoms attributable to $C$ difficile infection, of whom 12 were severely neutropenic. These included watery diarrhoea with abdominal pain in seven, mucous diarrhoea in four, and one child was critically ill and toxic as well as having diarrhoea. Of the seven children with an absolute neutrophil count greater than $0.5 \times 10^{9} / 1$, four had watery diarrhoea with abdominal pain and three had mucous stool in addition. The mean (SD) duration of symptoms was 6 (4) days with a range of 1-14 days. These children were infected with either $B, E$, $\mathrm{X}$, or $\mathrm{Z}$ strains, known to be highly pathogenic and toxigenic. ${ }^{3411}$ Nine children, seven of whom were severely neutropenic, had only mild symptoms and all were infected with $C$ difficile type $\mathrm{Y}$ non-toxigenic strains. ${ }^{10}$ 
Table 2 shows the antimicrobial treatment used. We had not developed specific guidelines for treatment so the $\operatorname{drug}(\mathrm{s})$ used and method of administration were chosen on the basis of the patients' condition alone. Nine children were symptomatic for less than 48 hours and received no treatment. Duration of treatment was for a minimum of five and a maximum of seven days.

The chemotherapeutic agents most frequently associated with $C$ difficile infection were the antileukaemic agents, a reflection of the most common diagnostic category in those admitted long term. The antibiotics most frequently implicated were the penicillins, cephalosporins, and aminoglycosides. However, this is the combination of antibiotics used in the management of all children with febrile neutropenia in our unit.

\section{Discussion}

The importance of typing is illustrated by the temporal distribution of cases in our study (figure), which was similar to that previously described $^{5}$ and would have suggested an outbreak of $C$ difficile. Typing proved that this was not the case. In addition type $\mathrm{X}$, previously shown to be highly infectious and responsible for an outbreak in an adult oncology ward, ${ }^{3}$ was seen in only three, albeit successive, cases.

The source of infection is not clear in our patients. The inpatient carriage rate of $C$ difficile is reported to vary from $3-4 \%$ on wards where there is no $C$ difficile associated diarrhoea to $15-30 \%$ on wards where there are patients with diarrhoea caused by $C$ difficile. ${ }^{611-13}$ In many cases the nosocomial acquisition of infection has been attributed to the introduction of a strain to the ward by an asymptomatic carrier. ${ }^{11-13}$ Indeed, $20-36 \%$ of paediatric cancer patients were reported to be carriers in one study and younger children appeared to be more frequently infected. ${ }^{6}$ In this study, no patient without a prior history of inpatient care (or admission to) in our unit was found to have $C$ difficile infection nor was age a determinant for infection. This differs from the previously mentioned study. Therefore in our study, while the incidence of $C$ difficile is comparable with other hospital based studies, the source of infection is more likely to be endogenous or environmental, ${ }^{14}$ although it is possible that some cross infection may have occurred with types $\mathrm{B}, \mathrm{X}$, and $\mathrm{E}$ (figure) between patients.

Isolation of $C$ difficile appeared to positively correlate with the duration of hospitalisation, a known risk factor. ${ }^{15}$ Children admitted for a short time on the same ward had a minimal risk of infection. Among those admitted for a longer duration, $22 \%$ were infected. Therefore, in our experience $C$ difficile does not appear to be as highly infectious as has previously been reported. ${ }^{6}$

As has been reported for adults with cancer, ${ }^{3}$ children with a diagnosis of acute leukaemia or lymphoma appear to be at significantly higher risk of infection. It is not possible from our study to identify the precise reason for this increase in susceptibility. However, of the 18 children with leukaemia or lymphoma, 15 were infected within the first two months after starting treatment, during and immediately after induction. Two other children were infected while being reinduced for a relapse and one child was found to be infected immediately after the first consolidation block (week 6 of treatment). Therefore the time of infection correlates with the periods of most marked and prolonged myelosuppression. This is the group of children with cancer that spends the longest time in hospital, receives the most myeloablative treatment and consequently is most frequently on antibiotics. Present day chemotherapy results in prolonged neutropenia, mucositis, and gut stasis creating a favourable milieu for $C$ difficile. Opportunistic proliferation can then be triggered by the use of an antibiotic regimen that selectively inhibits competitive colonic flora.

Nineteen of the 28 infected children had symptoms attributable to infection with $C$ difficile, the others having only very mild illnesses. The presence or absence of symptoms correlated with the type of $C$ difficile but not with the degree of neutropenia. Only one child was seriously ill, but it is not possible to categorically state that this was entirely due to $C$ difficile infection. In all the patients treated, symptoms resolved quickly and the organism was not isolated in specimens cultured immediately after treatment. One of 11 children who were treated and four of 17 children who received no treatment had a recurrence of infection. Unlike previous reports ${ }^{256}$ recurrence rates were low.

In our experience and that of others oral metronidazole or oral vancomycin appear to be equally effective in controlling symptoms and excretion. ${ }^{5} 613$ 16-18 Vancomycin has the theoretical advantage that, unlike metronidazole, when given orally it is not absorbed and therefore can attain significant bactericidal concentrations in the large intestine. ${ }^{2}$ However, it is at least 50 times as expensive and its use has been associated with the emergence of glycopeptide resistant Gram positive organisms. ${ }^{19}$ In those unable to take oral medications, intravenous metronidazole is the drug of choice as, unlike vancomycin, it is excreted into the gut when administered parenterally.

While treatment remains contentious and costly, prevention of outbreaks is simple and inexpensive. In our unit, patients with diarrhoea known or suspected to be due to $C$ difficile were nursed in cubicles but not barrier nursed, though barrier nursing is probably required for the control of an outbreak. ${ }^{4}$ One cannot too strongly emphasise the necessity for strict standards of hygiene. Ungloved hands frequently become contaminated with $C$ difficile during patient contact. ${ }^{15}$ Hand washing with soap and water do not reliably remove the organism and the wearing of gloves is recommended. ${ }^{20}$ Indirect evidence for this in our ward was that none of the highly susceptible patients undergoing autologous bone marrow rescue were infected. While the same medical and nursing team looks after all patients, 
reverse barrier nursing is strictly observed for those receiving megatherapy. The use of antimotility drugs such as loperamide is to be condemned. These drugs contribute to bowel stasis, proliferation of the organism, and increased toxicity and production of the cytocidal toxin. ${ }^{21}$ This can be a recipe for disaster in a child with chemotherapy induced toxic mucositis.

Our experience suggests that while $C$ difficile is an important pathogen in children with lymphoid malignancies, most patients have mild or no attributable symptoms. We speculate that in most cases, the organism is probably endogenous and is provided with a favourable milieu by the combination of cancer chemotherapy and broad spectrum antibiotics so it is difficult at times to distinguish those who are 'infected with' from those who are just 'excreting' the organism. While it is a nosocomial communicable disease, outbreaks are only likely with some pathogenic strains, for example, strain X. Treatment provides symptomatic relief and the rate of recurrence of infection is low (14\%). Early identification of cases and prompt institution of control measures will prevent possible outbreaks. It is important therefore that diarrhoea is not accepted as a normal symptom of cancer chemotherapy and stool specimens are sent for full bacteriological and viral investigation.

This work was supported in part by a Leonora Knatchbull paediatric oncology fellowship (VS) as well as a Leukaemia Research Fund fellowship (IS).

1 Saha V, Eden T. An odyssey in search of a cure - the treatment of acute lymphoblastic leukaemia in the UK. Indian f Pediatr 1993; 60: 525-38.

2 Bartlett JG. Antibiotic associated diarrhoea. Clin Infect Dis 1992; 15: 573-81.

3 Heard SR, Wren B, Barnett MJ, Thomas JM, Tabaqchali $\mathrm{S}$. Clostridium difficile infection in patients with haematological malignant disease. Epidemiol Infect 1988; 100: 63-72.

4 Heard SR, O'Farrell S, Holland D, Crook S, Barnett MJ, Tabaqchali $S$. The epidemiology of Clostridium difficile with the use of a typing scheme: nosocomial acquisition and cross-infection among immunocompromised patients. F Infect Dis 1986; 153: 3159-62.

5 Brunetto AL, Pearson ADJ, Craft AW, Pedler SJ. Clostridium difficile in an oncology unit. Arch Dis Child 1988; 63: 979-81.

6 Kelly CK, Pothoulakis C, LaMont JT. Clostridium colitis NEngl ₹ Med 1994; 330: 257-62.

7 Oskarsdóttir S, Mellander L, Márky I. Clostridium difficile in children with malignant disease. Pediatr Hematol Oncol in children with mali

8 George WL, Sutter VL, Citron D, Finegold SM. Selective and differential medium for isolation of Clostridium difficile. F Clin Microbiol 1979; 9: 214-9. 9 Tabaqchali S, O'Farrell S, Holland S, Silman R. Typing and epidemiological studies. Lancet 1984; i: 935-8.

10 Tabaqchali S, Holland D, O'Farrell S, Silman R. Method for typing of Clostridium difficile: based on polyacrylamide gel electrophoresis of ${ }^{35} \mathrm{~S}$ methionine labelled proteins. F Clin Microbiol 1986; 23: 197-8.

11 Wren B, Heard SR, Tabaqchali S. Association between production of toxins $A$ and $B$ and types of Clostridium production of toxins A and B and types
difficile. F Clin Pathol 1987; 40: 1397-401.

12 Clabot CR, Johnson S, Olson MM, Peterson LR, Gerding DN. Acquisition of Clostridium difficile by hospitalized patients: evidence for colonized new admissions as a patients: evidence for colonized new admission
source of infection. F Infect Dis 1992; 166: 561-7.

13 Bender BS, Bennet R, Laughon BE, et al. Is Clostridium difficile endemic in chronic care facilities? Lancet 1986; ii: 11-3

14 Wilcox MH, Spencer RC. Clostridium difficile infection: responses, relapses, re-infections. $\mathcal{f}$ Hosp Infect 1992; 22 85-92.

15 Burden DW. Clostridium difficile: the epidemiology and prevention of hospital-acquired infection. Infection 1982; 10: 203-4.

16 McFarland LV, Mulligan ME, Kwok RYY, Stamm WE Nosocomial acquisition of Clostridium difficile infection. N Engl f Med 1989; 321: 204-10

NEngl f Med 1989; 321: 204-10.
17 Walters BAJ, Roberts RK, Stafford R, Seneviratne E. Relapse of antibiotic associated colitis: endogenous persistence of Clostridium difficile during vancomycin therapy. Gut 1983; 24: 206-12.

18 O'Neill GL, Beaman MH, Riley TV. Relapse versus reinfection with Clostridium difficile. Epidemiol Infect 1991; 107: 627-35.

19 Saha V, Gupta S, Daum RS. Glycopeptide resistance in Gram positive cocci. Infectious Agents and Disease 1992; 6 310-8.

20 Johnson S, Gerding DN, Olson MM, et al. Prospective controlled study of vinyl glove use to interrupt Clostridium difficile nosocomial transmission. Am f Med 1990; 88: 137-9.

21 Klainer AS. Clindamycin. Med Clin North Am 1987; 71: 1169-75. 